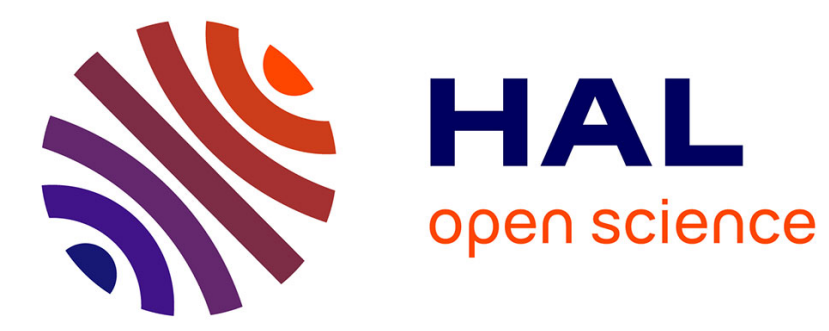

\title{
Breaking the resilience of a two-dimensional Bose-Einstein condensate to fragmentation
}

Shachar Klaiman, Axel Lode, Alexej Streltsov, Lorenz Cederbaum, Ofir Alon

\section{To cite this version:}

Shachar Klaiman, Axel Lode, Alexej Streltsov, Lorenz Cederbaum, Ofir Alon. Breaking the resilience of a two-dimensional Bose-Einstein condensate to fragmentation. Physical Review A, 2014, 90, pp.043620. 10.1103/PhysRevA.90.043620 . hal-02369826

\section{HAL Id: hal-02369826 \\ https://hal.science/hal-02369826}

Submitted on 19 Nov 2019

HAL is a multi-disciplinary open access archive for the deposit and dissemination of scientific research documents, whether they are published or not. The documents may come from teaching and research institutions in France or abroad, or from public or private research centers.
L'archive ouverte pluridisciplinaire HAL, est destinée au dépôt et à la diffusion de documents scientifiques de niveau recherche, publiés ou non, émanant des établissements d'enseignement et de recherche français ou étrangers, des laboratoires publics ou privés. 


\title{
Breaking the resilience of a two-dimensional Bose-Einstein condensate to fragmentation
}

\author{
Shachar Klaiman, ${ }^{1, *}$ Axel U. J. Lode, ${ }^{2}$ Alexej I. Streltsov, ${ }^{1}$ Lorenz S. Cederbaum, ${ }^{1}$ and Ofir E. Alon ${ }^{3}$ \\ ${ }^{1}$ Theoretische Chemie, Physikalisch-Chemisches Institut, Universität Heidelberg, Im Neuenheimer Feld 229, D-69120 Heidelberg, Germany \\ ${ }^{2}$ Department of Physics, University of Basel, Klingelbergstrasse 82, CH-4056 Basel, Switzerland \\ ${ }^{3}$ Department of Physics, University of Haifa at Oranim, Tivon 36006, Israel
}

(Received 11 July 2014; published 20 October 2014)

\begin{abstract}
A two-dimensional Bose-Einstein condensate (BEC) split by a radial potential barrier is investigated. We determine on an accurate many-body level the system's ground-state phase diagram as well as a time-dependent phase diagram of the splitting process. Whereas the ground state is condensed for a wide range of parameters, the time-dependent splitting process leads to substantial fragmentation. We demonstrate the dynamical fragmentation of a BEC despite its ground state being condensed. The results are analyzed using a mean-field model and suggest that a large manifold of low-lying fragmented excited states can significantly impact the dynamics of trapped two-dimensional BECs.
\end{abstract}

DOI: 10.1103/PhysRevA.90.043620

PACS number(s): 03.75.Kk, 03.65.-w, 05.30.Jp

\section{INTRODUCTION}

Shortly after the first experimental demonstration of trapped Bose-Einstein condensates (BECs) in three dimensions [1-3], BECs in two-dimensional traps have been realized [4-6]. While three-dimensional trapped BECs have been extensively studied since their discovery, the static and time-dependent properties of their two-dimensional counterparts are comparatively less explored.

One of the most popular scenarios studied with ultracold bosonic atoms, both experimentally and theoretically, is the splitting of a BEC by a central barrier into two spatially disjoint clouds (e.g., Refs. [7-21]). It is common knowledge that in order to produce a fragmented BEC in the splitting process, the ground state must be fragmented. This renders high barriers and strong interaction strengths necessary. Previous works dealt with splitting a BEC in one or three spatial dimensions. To the best of our knowledge, splitting a BEC in two spatial dimensions has not been explored experimentally or theoretically on the many-body level.

In the present work we investigate theoretically, on an accurate many-body level, the physics of splitting a twodimensional (2D) BEC. A natural approach is to exploit the 2D symmetry of the system. We thus split a circular BEC by a radial potential barrier [see Fig. 1(a) for an illustration]. This leads to two concentric clouds, unlike the above-discussed common way of splitting a BEC, and, as we shall see below, enriches the physics of BEC splitting.

By analyzing the many-body time-independent and timedependent wave functions of the system, we construct both static and dynamic phase diagrams of the splitting process. Whereas the ground state is condensed for a wide range of parameters, the time-dependent splitting process leads to substantial fragmentation. We therefore demonstrate the dynamical fragmentation of a BEC, despite its ground state being fully condensed. The results imply that a large manifold of fragmented excited states can significantly impact the dynamics of 2D BECs.

*Corresponding author: Shachar.Klaiman@pci.uni-heidelberg.de

\section{SETUP AND STATICS}

We consider a repulsive BEC with $N=100$ bosons in the 2D circular trap shown in Fig. 1(a). Throughout this work dimensionless units are used, such that the singleparticle kinetic-energy operator reads $\hat{T}(\mathbf{r})=-\frac{1}{2} \nabla_{\mathbf{r}}^{2}$ [22]. The explicit form of the one-body potential is given by $V(\mathbf{r})=$ $V_{\text {trap }}(\mathbf{r})+V_{\text {barrier }}(\mathbf{r})$. Here $V_{\text {trap }}(\mathbf{r})=\left\{200 e^{-\left(r-r_{c}\right)^{4} / 2}, r \leqslant r_{c}=\right.$ $\left.9 ; 200, r>r_{c}\right\}$ is a flat trap which has the shape of "a crater," and $V_{\text {barrier }}(\mathbf{r})=200 e^{-2(r-R)^{4}}$ is a ring-shaped radial barrier of radius $R$. We have chosen a flat potential $V_{\text {trap }}(\mathbf{r})$ in order to allow the BEC to fill in the full area.

\section{A. Noninteracting bosons}

It is instructive to commence with an analysis of the ground state of the noninteracting system. The single-particle Schrödinger equation reads $[\hat{T}(\mathbf{r})+V(\mathbf{r})] f(\mathbf{r})=\varepsilon f(\mathbf{r})$. The potential $V(\mathbf{r})$ can be considered to be made of two separated parts: an inner disk and an outer annulus [see Figs. 1(b) and 1(c)] separated by a radial barrier centered at $r=R$. Obviously, the energy $\varepsilon$ of the particle changes with the barrier's radius $R$. The energy of a particle in a disk of radius $R_{d}$ is well known and is given by $\varepsilon_{\text {disk }}=\frac{j_{0}^{2}}{2 R_{d}^{2}}$, where $j_{0}=2.4048$ is the first zero of the zeroth Bessel function (see, e.g., Ref. [23]). For an annulus of radii $R_{a 1}<R_{a 2}$, a remarkably precise (for not too small radii ratios) closedform expression has recently been given in [24] and reads $\varepsilon_{\text {annulus }} \approx \frac{\ln ^{2}\left(R_{a 1} / R_{a 2}\right)+\pi^{2}}{\left(R_{a 1}^{2}-R_{a 1}^{2}\right) \ln \left(R_{a 1} / R_{a 2}\right)}$. These expressions allow us to determine, as a function of $R$, where in the trap $V(\mathbf{r})$ the particle is located. For a high barrier, $\varepsilon_{\text {disk }}<\varepsilon_{\text {annulus }}$ implies that the particle is located in the inner disk, whereas the inverse relation $\varepsilon_{\text {annulus }}<\varepsilon_{\text {disk }}$ implies that it is localized in the outer annulus. Beyond the obvious effect of the size of each part of the trap dictated by the radius $R$, in $2 \mathrm{D}$ one must also consider the naturally occurring attractive term originating from the kinetic energy. Since the ground state is radially symmetric, $f(\mathbf{r})=f(r)$, making the standard change of variables $f(r) \rightarrow$ $\frac{f(r)}{\sqrt{r}}$, one finds $\left[-\frac{1}{2} \frac{\partial^{2}}{\partial r^{2}}+V(r)-\frac{1}{8} \frac{1}{r^{2}}\right] f(r)=\varepsilon f(r)$. Thus, for the ground state there is an effective attractive potential, $V_{2 D}(r)=-\frac{1}{8} \frac{1}{r^{2}}$, pulling the particle towards the center. This 
(a)

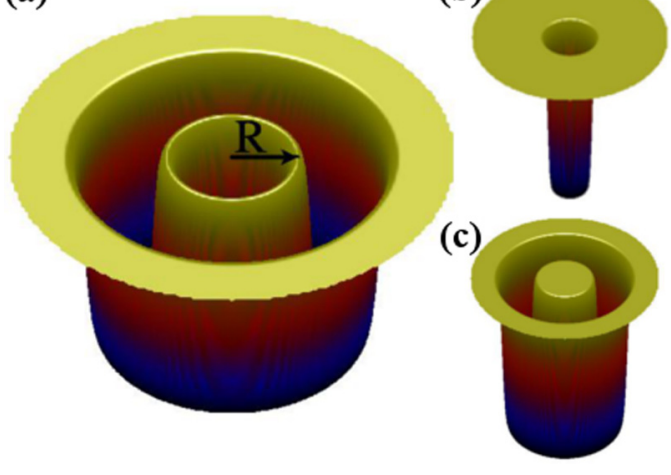

FIG. 1. (Color online) (a) The two-dimensional circular trap split by a radial barrier of radius $R$. (b) The inner-disk and (c) outer-annulus model potentials used to interpret the ground-state phase diagram.

attractive force plays a crucial role in the physics described below. Furthermore, one might expect that there is a critical $R$ for which $\varepsilon_{\text {disk }}=\varepsilon_{\text {annulus }}$ and the particle is located both in the disk and the annulus parts of the trap. We will return to these points when the interaction is turned on and will offer a generalization thereof.

\section{B. Interacting bosons}

We now switch on the interaction between the particles and move to investigate its effect on the ground state of the system. Specifically, we would like to study the one-body coherence properties of the ground state and ascertain when the many-body state is fragmented [25-35] or condensed [36]. This many-body property, which is derived from the eigenvalues of the reduced one-body density matrix [37,38], unambiguously conveys whether the BEC can be described within a single-orbital mean-field theory, i.e., the GrossPitaevskii (GP) equation, or it is necessary to solve for the many-body state which spreads the bosons over many orbitals. Clearly, one can know this only a posteriori, so we must solve the complicated many-body Hamiltonian in order to know whether the GP equation would have sufficed.

A suitable platform to study the many-body time evolution of trapped BECs is provided by the multiconfigurational timedependent Hartree for bosons (MCTDHB) method [18,39]. The MCTDHB method has been shown to produce accurate many-body solutions in various applications [19,40-44] and is well documented in the literature [45,46] (also see the Appendix). Until recently, MCTDHB has been applied to one-dimensional systems. Most recently, MCTDHB has been implemented in higher dimensions [44], which allows us now to enlarge the range of applications to two and three dimensions. We use the implementation in the recursive MCTDHB (R-MCTDHB) [47] and MCTDHB [48] software packages.

The many-boson Hamiltonian is given by $\hat{H}\left(\mathbf{r}_{1}, \ldots, \mathbf{r}_{N}\right)=$ $\sum_{j=1}^{N}\left[\hat{T}\left(\mathbf{r}_{j}\right)+V\left(\mathbf{r}_{j}\right)\right]+\sum_{j<k} W\left(\mathbf{r}_{j}-\mathbf{r}_{k}\right)$. The short-range repulsive interaction between the bosons is modeled by a Gaussian function $[49,50] W\left(\mathbf{r}-\mathbf{r}^{\prime}\right)=\lambda_{0} \frac{e^{-\left(\mathbf{r}-\mathbf{r}^{\prime}\right)^{2} / 2 \sigma^{2}}}{2 \pi \sigma^{2}}$ with a width $\sigma=0.25$. The interaction parameter $\lambda_{0}$ is taken to be positive to describe repulsive bosons. A square box of size
$[-12,12) \times[-12,12)$ and spatial grid of size $128 \times 128$ were found to converge the results to the accuracy given below.

Let $\Psi\left(\mathbf{r}_{1}, \mathbf{r}_{2}, \ldots, \mathbf{r}_{N}\right)$ be the normalized many-body wave function of the system. The reduced onebody density matrix then reads $\rho\left(\mathbf{r}, \mathbf{r}^{\prime}\right)=N \int d \mathbf{r}_{2} \cdots$ $\int d \mathbf{r}_{N} \Psi^{*}\left(\mathbf{r}^{\prime}, \mathbf{r}_{2}, \ldots, \mathbf{r}_{N}\right) \Psi\left(\mathbf{r}, \mathbf{r}_{2}, \ldots, \mathbf{r}_{N}\right)$. Diagonalizing it, we have $\rho\left(\mathbf{r}, \mathbf{r}^{\prime}\right)=\sum_{j} n_{j} \psi_{j}^{*}\left(\mathbf{r}^{\prime}\right) \psi_{j}(\mathbf{r})$, where $n_{j}$ are referred to as the natural occupation numbers and $\psi_{j}(\mathbf{r})$ are the natural orbitals. It is customary to list the natural occupation numbers in nonincreasing order, i.e., $n_{1} \geqslant n_{2} \cdots$. In the present work, in order to quantify the fragmentation of the many-body state $\Psi$, it is convenient to define it as $\frac{1}{N} \sum_{j>1} n_{j}$.

Figure 2 depicts the ground-state fragmentation versus the position of the radial barrier for three different interaction strengths, $\lambda_{0}=0.002,0.02,0.2$ [51]. These many-body phase diagrams show that the radii $R$ for which the ground state is fragmented are very limited, that is, that the ground state is mostly condensed within the parameter space of the problem. Increasing the interaction strength leads to two distinct effects. First, the maximal fragmentation shifts to larger values of $R$, and second, the width of the fragmented region also increases with the interaction. Importantly, we note that essentially $50 \%$ fragmentation for different interaction strengths has been reached. The maxima occur for traps of different radii. Throughout this work we have performed all computations with four orbitals and have found that no more than two orbitals are macroscopically occupied (see the Appendix).

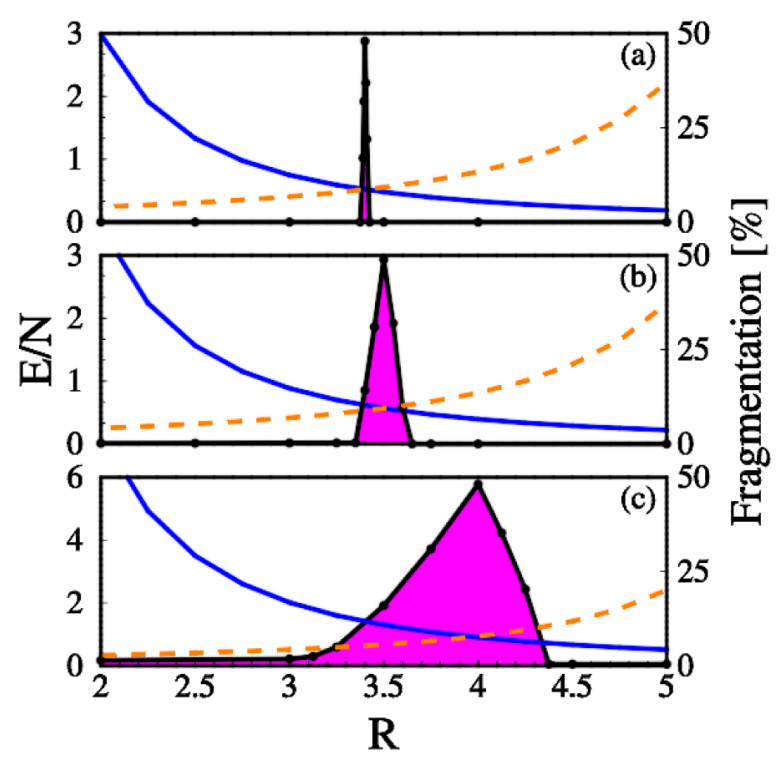

FIG. 2. (Color online) Ground-state many-body phase diagram of a BEC in a circular trap. The three panels correspond, from top to bottom, to the interaction strengths $\lambda_{0}=0.002,0.02$, and 0.2 . The number of bosons is $N=100$. The splitting changes the coherence properties of the BEC. The BEC is mostly condensed, except for a narrow window (magenta shaded area) of radii $R$, which depends on $\lambda_{0}$. The weaker the interaction is, the narrower the window in which the BEC is fragmented is. A static model based on the GP theory is shown. The energies $\varepsilon_{\text {disk }}^{\mathrm{GP}}$ (solid blue line) and $\varepsilon_{\text {annulus }}^{\mathrm{GP}}$ (dashed orange line) as a function of $R$ are depicted. The maximal fragmentation on the many-body level is encountered when $\varepsilon_{\text {disk }}^{\mathrm{GP}}=\varepsilon_{\text {annulus }}^{\mathrm{GP}}$. All quantities are dimensionless. 
Hence, the fragmentation of the BEC essentially equals the second eigenvalue of the reduced one-body density matrix.

In order to understand the phase diagrams depicted in Fig. 2, we set up a model. The model is based on the GP mean-field solutions of the $N$ interacting bosons in the inner-disk and the outer-annulus parts [see Figs. 1(b) and 1(c)]. The GP energies per particle, $\varepsilon_{\text {disk }}^{\mathrm{GP}}$ and $\varepsilon_{\text {annulus }}^{\mathrm{GP}}$, are depicted as a function of the barrier's position $R$ in Fig. 2 for each interaction strength. Remarkably, the intersection points of the two curves, $\varepsilon_{\text {disk }}^{\mathrm{GP}}=\varepsilon_{\text {annulus }}^{\mathrm{GP}}$, which mark a mean-field degeneracy between the inner and outer parts of the trap, accurately indicate the maximal fragmentation of the system on the many-body level. Moreover, the density of the fragmented ground state of the split BEC occupies both the inner and the outer parts of the trap (see Fig. 3). In the limit of weak interaction, our mean-field model connects with the noninteracting system discussed above. Namely, for the parameters of the potential studied, the noninteracting model predicts a degeneracy around $R=3.3$, which is in quite good agreement with the maximal fragmentation in the case of the weak interaction, i.e., $R=3.4$.

The results in Figs. 2 and 3 clearly indicate that fragmentation of the BEC is accompanied by the spatial occupation of both the inner disk and the outer annulus of the trap. When the interaction energy is larger than the energy difference between the disk and the annulus, the BEC spreads over the two parts. Consequently, the fragmented region in the phase diagram increases as the interaction becomes stronger. Within the fragmented region in the phase diagrams, the energy of the fragmented system is lower than the energy of the condensed system.
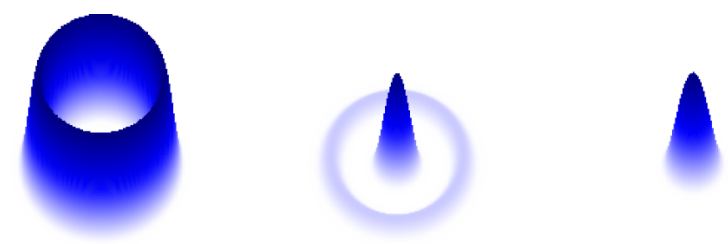

Ground state at $\mathrm{R}=3$ Ground state at $\mathrm{R}=3.5$ Ground state at $\mathrm{R}=4$
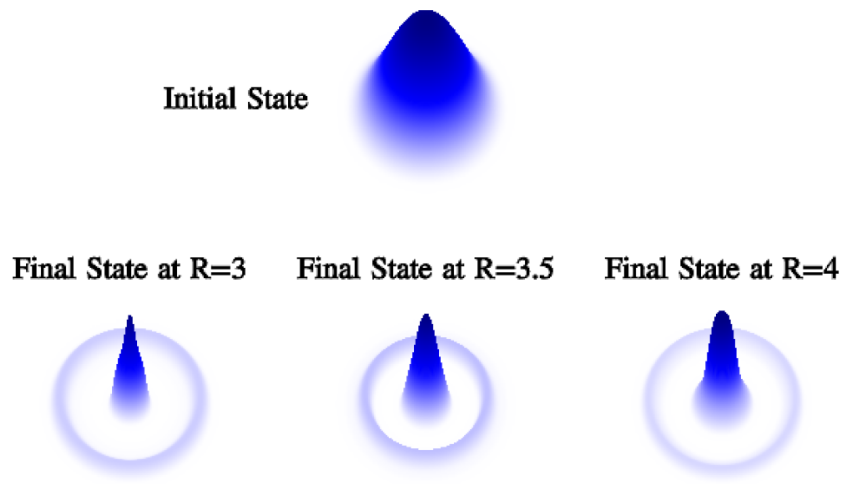

FIG. 3. (Color online) The densities of the ground state and of the wave function after the splitting process for $\lambda_{0}=0.02$. (top) For different values of $R$ the ground state is located either inside the inner-disk part, outside in the annular part, or in a combination of both. (middle) Without the radial barrier, the BEC is spread out in the circular trap $V_{\text {trap }}(\mathbf{r})$. (bottom) The density after the splitting process is located both in the disk and annulus for a wide range of radii $R$.
Another interesting property of the phase diagrams is that the radius $R$ where the fragmentation is maximal increases with $\lambda_{0}$. For the disk and annular regions to be energetically equivalent (in the GP sense), the disk part should be smaller because of the attraction $V_{2 D}(r)$ towards the center. Hence, the GP orbital in the disk is more localized than the GP orbital in the annulus. Since the interaction energy scales like the fourth power of the GP orbital, when $\lambda_{0}$ is enlarged, $R$ must increase in order to compensate for the growing interaction energy.

So far, we have explored the static properties of the ground state, showing it is mostly condensed. One might expect that dynamically splitting a BEC by raising a radial barrier would also lead to a condensed state, at the very least in the adiabatic limit when the radial barrier is raised slowly enough. It turns out that the dynamical picture is much more intriguing.

\section{OUT-OF-EQUILIBRIUM DYNAMICS}

To explore the dynamical process of splitting the BEC, we prepare the BEC in the ground state of the trap $V_{\text {trap }}(\mathbf{r})$. In the absence of the radial barrier, the BEC is spread in the flat circular trap (see Fig 3). One then ramps up the radial barrier such that the time-dependent one-body potential reads $V(\mathbf{r}, t)=$ $V_{\text {trap }}(\mathbf{r})+V_{\text {ramp-up }}(\mathbf{r}, t)$, where $V_{\text {ramp-up }}(\mathbf{r}, t)=\frac{\beta t}{200} V_{\text {barrier }}(\mathbf{r})$ and $\beta$ is the splitting rate (the ramp-up process stops when the barrier reaches its maximal height, i.e., $\beta t=200)$. This is a demanding many-body problem in $2 \mathrm{D}$ because the BEC significantly changes both its shape and coherence, which MCTDHB can efficiently handle $[47,48]$.

Figure 4(a) depicts the fragmentation at the end of the splitting process for interaction strength $\lambda_{0}=0.02$ as a function of the radius $R$. The splitting rate is $\beta=1$. The dynamical splitting process leads to fragmentation over the entire examined range of radii $R$. For most of these radii the ground state of the system (at any barrier height) is condensed. The system can thus dynamically fragment even though the ground state is condensed. Compared to the static phase diagram [Fig. 2(b)], the regime of dynamical fragmentation of the 2D BEC is significantly larger.

Regardless of whether the ground-state density is located in the inner disk, the outer annulus, or in both parts of the trap, the fragmented final state is spread over the entire trap (see Fig. 3). This generic feature can be understood from the two opposing forces acting on the BEC. On the one hand, the $2 \mathrm{D}$ attractive term $V_{2 D}(r)$ tends to localize the particles in the inner disk. The repulsive interaction, on the other hand, naturally tends to push them apart from one another. This competition promotes the dynamical spread of the BEC over the inner disk and the outer annulus in the splitting process.

To test the generality of the obtained results we have also considered additional particle numbers, explicitly $N=20$ and $N=50$ bosons. We have repeated the statics investigations (not shown) and, subsequently, the dynamical investigations, which are collected in Fig. 5. Indeed, the same results are obtained for $N=20$ and $N=50$ bosons as well; that is, the range of dynamical fragmentation is substantially broader than ground-state fragmentation.

To have a broader picture of the physical process we also study the fragmentation of the system as a function of the splitting rate $\beta$. Choosing a radius for which the ground state 

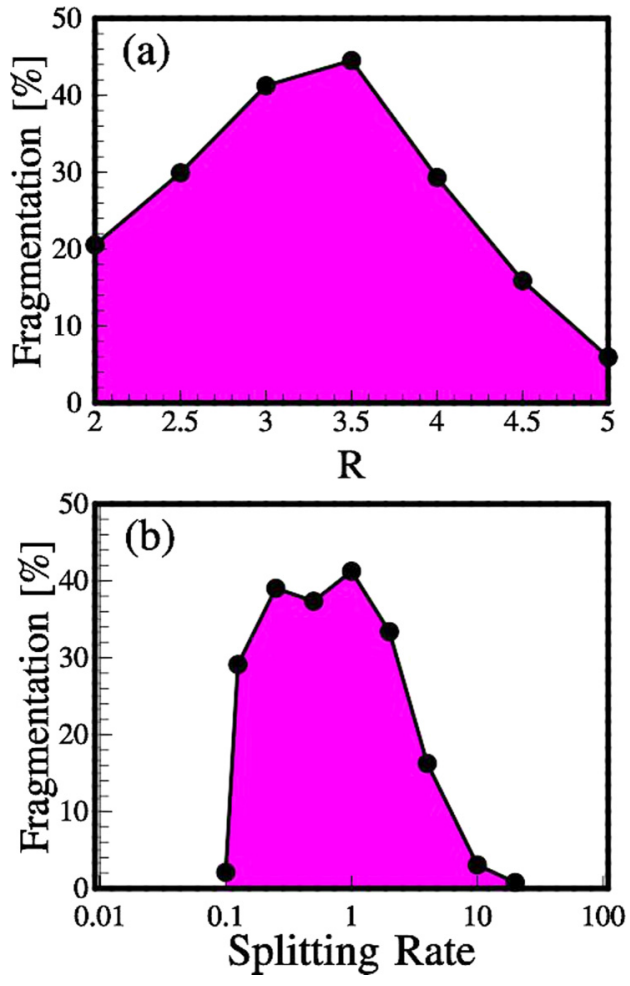

FIG. 4. (Color online) Two cuts through the time-dependent phase diagram for $N=100$ bosons and interaction strength $\lambda_{0}=$ 0.02 . The fragmentation depicted refers to the end of the splitting process. (a) Remarkably, the dynamical splitting process leads to fragmentation over the entire examined range of radii $R$; for comparison, see Fig. 2(b). The splitting rate is $\beta=1$. (b) The dynamical splitting process leads to fragmentation over two orders of magnitude of the splitting rate. The radius is $R=3$. In the splitting process the system has a high affinity to fragment. All quantities are dimensionless.

is condensed, $R=3$, we varied $\beta$ over two orders of magnitude [see Fig. 4(b)]. This cut through the dynamical phase diagram produces a broad region of splitting rates in which the system dynamically fragments. For slow rates $(\beta<0.1)$ the system remains condensed throughout the splitting process.

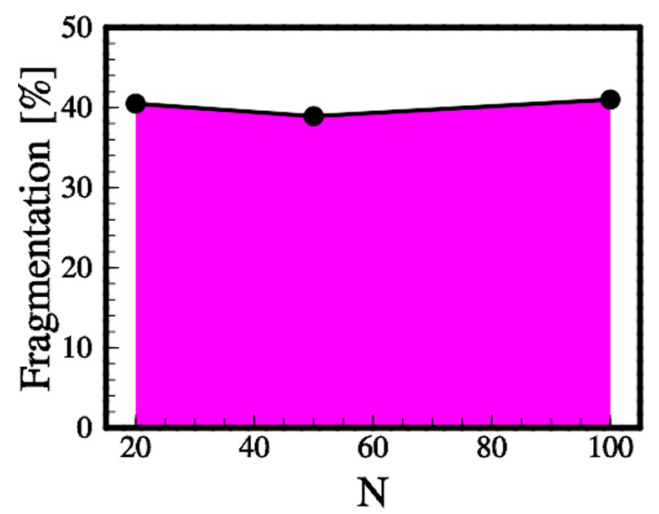

FIG. 5. (Color online) Same as Fig. 4(a) at barrier radius $R=$ 3 but for different particle numbers $N$. The interaction parameter $\lambda_{0}(N-1)=2$ is kept fixed. All quantities are dimensionless.
Interestingly, for fast rates $(\beta>10)$ the system also remains condensed. This suggests that the system requires a finite amount of time in order to fragment and that pumping more energy into the system does not necessarily lead to larger fragmentation. In between these two regions, the dynamical splitting process leads to fragmentation over two orders of magnitude of $\beta$.

Fragmentation involves transferring bosons out of the condensed mode. This means that the condensed initial state must overlap with a manifold of excited states with successively increasing degrees of fragmentation. If the splitting process is too slow, the system does not reach these states, whereas if it is too fast, there is no time to efficiently go through such a manifold of fragmented excited states.

\section{CONCLUDING REMARKS}

In conclusion, the present research investigated the manybody physics of splitting a $2 \mathrm{D}$ BEC by a radial barrier. We determined the static phase diagram, which demonstrated the resilience of a $2 \mathrm{D}$ BEC to fragmentation. The ground state can only fragment in the vicinity of the degeneracy of GP energy of the two parts of the potential. The position of this degeneracy and the width of the fragmented region depend on the interaction strength. We then explored the dynamical process of splitting the BEC by a time-dependent barrier. This yielded a dynamic phase diagram, which revealed that the system fragments over a much larger region compared to the static results. Strikingly, the dynamical fragmentation of a BEC, despite its ground state being fully condensed, was thus identified. This opens up exciting possibilities beyond the current practice; that in order to produce a fragmented BEC in the splitting process, the ground state must be fragmented. Furthermore, our study suggests that a large manifold of fragmented excitations can significantly impact the dynamics of trapped 2D BECs.

As an outlook, we mention that implementing many-body linear response in 2D would provide the low-lying excitations, which are not recovered using standard methods [52]. This

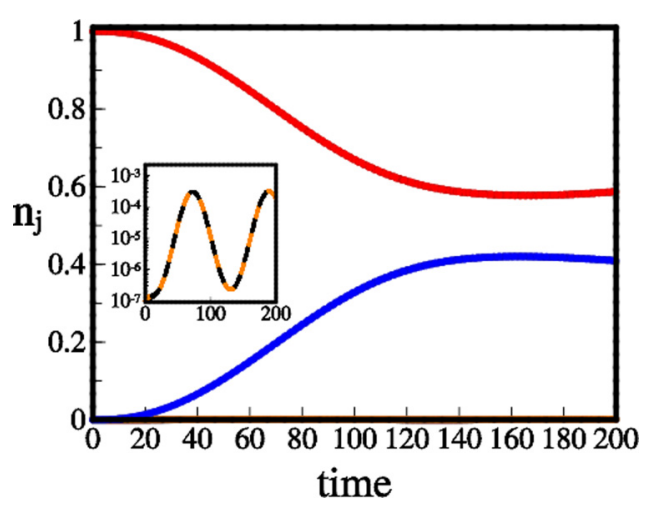

FIG. 6. (Color online) Accuracy of the MCTDHB computations. Time evolution of the natural occupation numbers $n_{j}$ (divided by the number of bosons $N$ ) for the split dynamics of Fig. 4(a) at barrier radius $R=3$. No more than two orbitals are macroscopically occupied. The inset shows the occupation of the third and fourth natural orbitals. All quantities are dimensionless. 
would shed further light on the present findings. We also speculate that the effect of dynamical fragmentation could be relevant in other circularly shaped setups, such as those in Ref. [53]. We believe the present work will stimulate the experimental and theoretical exploration of many-body dynamics in these systems.

\section{ACKNOWLEDGMENTS}

Financial support from the DFG is gratefully acknowledged. A.U.J.L. acknowledges financial support from the Swiss SNF and the NCCR Quantum Science and Technology. Computation time on the Cray XE6 system Hermit and the NEC Nehalem cluster Laki at the HLRS and on the bwGRiD cluster is gratefully acknowledged.

\section{APPENDIX: ACCURACY OF THE MCTDHB COMPUTATIONS}

The MCTDHB method [18,39] and software packages $[47,48]$ are based on the real-space Hamiltonian $\hat{H}\left(\mathbf{r}_{1}, \ldots, \mathbf{r}_{N}\right)$. MCTDHB uses time-dependent orbitals which are determined using the variational principle. Numerically, the integration scheme employs an adaptive time step. Utilizing time-dependent orbitals allows one to obtain accurate numerical results with substantially less numerical resources than a corresponding computation with fixed orbitals would need. The accuracy of the method has recently been benchmarked (see [41]).

As a concrete example and without loss of generality, the time evolution of the natural occupation numbers $n_{j}$ for the split dynamics in Fig. 4(a) at barrier radius $R=3$ is shown in Fig. 6. As can be seen, no more than two orbitals are macroscopically occupied.
[1] M. H. Anderson, J. R. Ensher, M. R. Matthews, C. E. Wieman, and E. A. Cornell, Science 269, 198 (1995).

[2] C. C. Bradley, C. A. Sackett, J. J. Tollett, and R. G. Hulet, Phys. Rev. Lett. 75, 1687 (1995).

[3] K. B. Davis, M.-O. Mewes, M. R. Andrews, N. J. van Druten, D. S. Durfee, D. M. Kurn, and W. Ketterle, Phys. Rev. Lett. 75, 3969 (1995).

[4] A. Görlitz, J. M. Vogels, A. E. Leanhardt, C. Raman, T. L. Gustavson, J. R. Abo-Shaeer, A. P. Chikkatur, S. Gupta, S. Inouye, T. Rosenband, and W. Ketterle, Phys. Rev. Lett. 87, 130402 (2001).

[5] D. Rychtarik, B. Engeser, H.-C. Nägerl, and R. Grimm, Phys. Rev. Lett. 92, 173003 (2004).

[6] I. Bloch, J. Dalibard, and W. Zwerger, Rev. Mod. Phys. 80, 885 (2008).

[7] A. J. Leggett and F. Sols, Phys. Rev. Lett. 81, 1344 (1998).

[8] J. Javanainen and M. Yu. Ivanov, Phys. Rev. A 60, 2351 (1999).

[9] C. Menotti, J. R. Anglin, J. I. Cirac, and P. Zoller, Phys. Rev. A 63, 023601 (2001).

[10] Y. Shin, M. Saba, T. A. Pasquini, W. Ketterle, D. E. Pritchard, and A. E. Leanhardt, Phys. Rev. Lett. 92, 050405 (2004).

[11] M. Albiez, R. Gati, J. Fölling, S. Hunsmann, M. Cristiani, and M. K. Oberthaler, Phys. Rev. Lett. 95, 010402 (2005).

[12] L. Pezzé, L. A. Collins, A. Smerzi, G. P. Berman, and A. R. Bishop, Phys. Rev. A 72, 043612 (2005).

[13] T. Schumm, S. Hofferberth, L. M. Andersson, S. Wildermuth, S. Groth, I. Bar-Joseph, J. Schmiedmayer, and P. Krüger, Nat. Phys. 1, 57 (2005).

[14] A. Mebrahtu, A. Sanpera, and M. Lewenstein, Phys. Rev. A 73, 033601 (2006).

[15] L. Isella and J. Ruostekoski, Phys. Rev. A 74, 063625 (2006).

[16] B. V. Hall, S. Whitlock, R. Anderson, P. Hannaford, and A. I. Sidorov, Phys. Rev. Lett. 98, 030402 (2007).

[17] G.-B. Jo, Y. Shin, S. Will, T. A. Pasquini, M. Saba, W. Ketterle, D. E. Pritchard, M. Vengalattore, and M. Prentiss, Phys. Rev. Lett. 98, 030407 (2007).

[18] A. I. Streltsov, O. E. Alon, and L. S. Cederbaum, Phys. Rev. Lett. 99, 030402 (2007).

[19] J. Grond, J. Schmiedmayer, and U. Hohenester, Phys. Rev. A 79, 021603(R) (2009).
[20] D. K. Faust and W. P. Reinhardt, Phys. Rev. Lett. 105, 240404 (2010).

[21] E. Torrontegui, S. Martínez-Garaot, M. Modugno, Xi Chen, and J. G. Muga, Phys. Rev. A 87, 033630 (2013).

[22] To convert to dimensionless units the Hamiltonian is divided by $\frac{\hbar^{2}}{m L^{2}}$, where $m$ is the mass of a boson and $L$ is a length scale. As a concrete example, we choose $L=2 \mu \mathrm{m}$ and use the ${ }^{87} \mathrm{Rb}$ atom as a boson. We point out that other realistic choices can be made. The unit of energy $\frac{\hbar^{2}}{m L^{2}}$ corresponds to $29.1 \mathrm{~Hz}$, and the unit of time $\frac{m L^{2}}{\hbar}$ corresponds to $5.47 \times 10^{-3} \mathrm{~s}$. The trapping potential height is $V=5.82 \mathrm{kHz}$, and the radius of the crater is $r_{c}=18 \mu \mathrm{m}$.

[23] J. R. Kuttler and V. G. Sigillito, SIAM Rev. 26, 163 (1984).

[24] C. Alvarado and P. Amore, J. Math. Phys. 52, 063516 (2011).

[25] P. Nozières and D. Saint James, J. Phys. (Paris) 43, 1133 (1982).

[26] P. Nozières, in Bose-Einstein Condensation, edited by A. Griffin, D. W. Snoke, and S. Stringari (Cambridge University Press, Cambridge, 1996), p. 15.

[27] R. W. Spekkens and J. E. Sipe, Phys. Rev. A 59, 3868 (1999).

[28] A. I. Streltsov, O. E. Alon, and L. S. Cederbaum, Phys. Rev. A 73, 063626 (2006).

[29] E. J. Mueller, T.-L. Ho, M. Ueda, and G. Baym, Phys. Rev. A 74, 033612 (2006).

[30] P. Bader and U. R. Fischer, Phys. Rev. Lett. 103, 060402 (2009).

[31] U. R. Fischer and P. Bader, Phys. Rev. A 82, 013607 (2010).

[32] Q. Zhou and X. Cui, Phys. Rev. Lett. 110, 140407 (2013).

[33] Y. Kawaguchi, Phys. Rev. A 89, 033627 (2014).

[34] S.-W. Song, Y.-C. Zhang, H. Zhao, X. Wang, and W.-M. Liu, Phys. Rev. A 89, 063613 (2014).

[35] M.-K. Kang and U. R. Fischer, Phys. Rev. Lett. 113, 140404 (2014).

[36] O. Penrose and L. Onsager, Phys. Rev. 104, 576 (1956)

[37] P.-O. Löwdin, Phys. Rev. 97, 1474 (1955).

[38] A. Coleman and V. Yukalov, Reduced Density Matrices: Coulson's Challenge (Springer, Heidelberg, 2000).

[39] O. E. Alon, A. I. Streltsov, and L. S. Cederbaum, Phys. Rev. A 77, 033613 (2008).

[40] K. Sakmann, A. I. Streltsov, O. E. Alon, and L. S. Cederbaum, Phys. Rev. Lett. 103, 220601 (2009). 
[41] A. U. J. Lode, K. Sakmann, O. E. Alon, L. S. Cederbaum, and A. I. Streltsov, Phys. Rev. A 86, 063606 (2012).

[42] M. Heimsoth, D. Hochstuhl, C. E. Creffield, L. D. Carr, and F. Sols, New J. Phys. 15, 103006 (2013).

[43] A. I. Streltsov, Phys. Rev. A 88, 041602(R) (2013).

[44] O. I. Streltsova, O. E. Alon, L. S. Cederbaum, and A. I. Streltsov, Phys. Rev. A 89, 061602(R) (2014).

[45] Multidimensional Quantum Dynamics: MCTDH Theory and Applications, edited by H.-D. Meyer, F. Gatti, and G. A. Worth (Wiley-VCH, Weinheim, 2009).

[46] Quantum Gases: Finite Temperature and Non-Equilibrium Dynamics, edited by N. P. Proukakis, S. A. Gardiner, M. J. Davis, and M. H. Szymanska, Cold Atoms Series Vol. 1 (Imperial College Press, London, 2013).

[47] A. U. J. Lode and M. C. Tsatsos, The Recursive Multiconfigurational Time-Dependent Hartree for Bosons Package, version 1.0 (2014), http://ultracold.org.

[48] A. I. Streltsov, K. Sakmann, A. U. J. Lode, O. E. Alon, and L. S. Cederbaum, The Multiconfigurational Time-Dependent Hartree for Bosons Package, version 2.3 (2013), http://MCTDHB.org.
[49] J. Christensson, C. Forssén, S. Åberg, and S. M. Reimann, Phys. Rev. A 79, 012707 (2009)

[50] R. A. Doganov, S. Klaiman, O. E. Alon, A. I. Streltsov, and L. S. Cederbaum, Phys. Rev. A 87, 033631 (2013).

[51] The relation between the dimensionless interaction parameter $\lambda_{0}$ and the scattering length $a_{s}$ and the transverse confinement $l_{z}=\sqrt{\frac{\hbar}{m \omega_{z}}}$ along the $z$ axis is given by $\lambda_{0}=2 \sqrt{2 \pi} \frac{a_{s}}{l_{z}}$, where [54] is used. To realize the cases considered in this work for $N=100$ bosons, $a_{s}$ and $\omega_{z}$ can be chosen as follows. Let the aspect ratio be $\frac{l_{z}}{2 r_{c}}=\frac{1}{100}$, i.e., $\omega_{z}=2 \pi \times 897 \mathrm{~Hz}$. Then for $\lambda_{0}=0.002, a_{s}=0.144 \mathrm{~nm}$; for $\lambda_{0}=0.02, a_{s}=1.44 \mathrm{~nm}$; and for $\lambda_{0}=0.2, a_{s}=14.4 \mathrm{~nm}$.

[52] J. Grond, A. I. Streltsov, A. U. J. Lode, K. Sakmann, L. S. Cederbaum, and O. E. Alon, Phys. Rev. A 88, 023606 (2013).

[53] S. Eckel, J. G. Lee, F. Jendrzejewski, N. Murray, C. W. Clark, C. J. Lobb, W. D. Phillips, M. Edwards, and G. K. Campbell, Nature (London) 506, 200 (2014).

[54] D. S. Petrov, M. Holzmann, and G. V. Shlyapnikov, Phys. Rev. Lett. 84, 2551 (2000). 